\title{
Influence of geometrical parameters on the linear stability of a Bénard-Marangoni problem
}

\author{
S. Hoyas, ${ }^{1,}{ }^{*}$ P. Fajardo, ${ }^{2}$ and M. J. Pérez-Quiles ${ }^{1}$ \\ ${ }^{1}$ Instituto Universitario de Matemática Pura y Aplicada, Universitat Politècnica de València, València, Spain \\ ${ }^{2}$ Aerospace Engineering Group, Universidad Carlos III de Madrid, Leganés 28911, Spain \\ (Received 8 June 2015; revised manuscript received 11 February 2016; published 5 April 2016)
}

\begin{abstract}
A linear stability analysis of a thin liquid film flowing over a plate is performed. The analysis is performed in an annular domain when momentum diffusivity and thermal diffusivity are comparable (relatively low Prandtl number, $\operatorname{Pr}=1.2)$. The influence of the aspect ratio $(\Gamma)$ and gravity, through the Bond number $(\mathrm{Bo})$, in the linear stability of the flow are analyzed together. Two different regions in the $\Gamma$-Bo plane have been identified. In the first one the basic state presents a linear regime (in which the temperature gradient does not change sign with $r$ ). In the second one, the flow presents a nonlinear regime, also called return flow. A great diversity of bifurcations have been found just by changing the domain depth $d$. The results obtained in this work are in agreement with some reported experiments, and give a deeper insight into the effect of physical parameters on bifurcations.
\end{abstract}

DOI: 10.1103/PhysRevE.93.043105

\section{INTRODUCTION}

The study of the thermoconvective instabilities in fluid layers driven by a temperature gradient has been a subject in fluid mechanics $[1,2]$ for more than a century. When both gravity and capillary forces are taken into account, this problem is referred as a Bénard-Marangoni convection problem. Classically in this problem, heat is applied from below [1]. A more general setup may be considered which includes thermoconvective instabilities by imposing a basic dynamic flow through horizontal temperature gradients [3-5].

To characterize the different effects influencing the behavior of the flow, the following set of dimensionless numbers are typically used:

(1) The Rayleigh number $\mathrm{Ra}$ is representative of the buoyancy effect. It is the control parameter used in this work: $\mathrm{Ra}=g \alpha \Delta T d^{3} / \kappa \nu$.

(2) The Marangoni number Ma, which accounts for the surface tension effects: $\mathrm{Ma}=\gamma_{T} \Delta T d / \rho \kappa \nu$.

(3) The Prandtl number Pr, which is the ratio of momentum diffusivity to thermal diffusivity: $\operatorname{Pr}=\nu / \kappa$. In this paper a value of $\operatorname{Pr}=1.2$, which is a typical value for gases, is used. For this value the convective term of the Navier-Stokes equation cannot be neglected.

(4) The Biot number Bi accounts for heat transfer between the fluid and the atmosphere. This number has been fixed to 1 in this study, a value similar to the one in the experimental works of $[3,6]$.

In the previous definitions, $\gamma_{T}$ stands for the rate of change of surface tension with temperature; $\Delta T$ is the temperature increment; $\rho, \kappa, \alpha$, and $v$ are the density, the thermal diffusivity, the thermal expansion coefficient, and the kinematic viscosity of the fluid, respectively; and $g$ is the acceleration due to gravity.

The ratio between the Rayleigh and Marangoni numbers, also known as the Bond number, Bo $=\frac{\rho g \alpha d^{2}}{\gamma_{T}}$, depends on the domain geometry and the gravity $g$, which is modified in this work to analyze its influence on the flow instabilities. It is convenient to mention that the Bond number used is this

\footnotetext{
*serhocal@mot.upv.es
}

work is the thermal Bond number defined as the ratio between thermogravitational effects and thermocapillary effects, and should not be mistaken with the Bond number commonly used in interfacial systems. In most of the cases studied in this paper, Bo $\gg 1$ and thus buoyancy effects are dominant.

The onset of instabilities in thermoconvective problems has been analyzed in [4,5]. These instabilities have been previously studied both in rectangular domains containing the flow $[2,7,8]$ and in annular geometries [9-11]. More recently, the problem was also assessed in an annular geometry $[12,13]$ but neglecting the effect of Biot number, i.e., the heat transfer between the flow and the atmosphere through the upper surface of the domain, and considering conduction through the lateral walls of the cylinder. Hoyas et al. [6] studied the influence of Biot number on the competing solutions for the case of $\operatorname{Pr}=\infty$ and the computational results were validated against the experimental results by Garnier et al. [3]. The computational procedure has been extended for Prandtl numbers close to unity [14].

In [10], the authors investigated the existence of codimension-three bifurcations that are the points where the codimensions two curves intersect on the Prandtl-Biot plane and an also new kind of instability was found, hydrothermal waves of second kind (HWII). The interest in understanding the influence of gravitational effects in thermoconvective phenomena has been rapidly growing [15-17]. However, less attention has been paid to the effect of the domain geometry on the onset of the flow motion and the dynamics of the different bifurcations appearing.

The current work is devoted to obtain a deeper insight on the effect of the domain geometry on the development of flow instabilities. The understanding of this flow behavior will contribute to open a gateway to control these instabilities. To achieve this goal, a linear stability analysis, similar to the one in [6], will be performed, but instead of focusing on the influence of the Prandtl number, the focus will be put on understanding the effect of the domain geometry, characterized by the aspect ratio (a complete definition of the aspect ratio will be presented in the following section).

The paper is structured as follows. In the second section the formulation of the problem is presented. The third one describes the numerical method used to obtain the solution. Then, in the fourth section the results are discussed. In the 


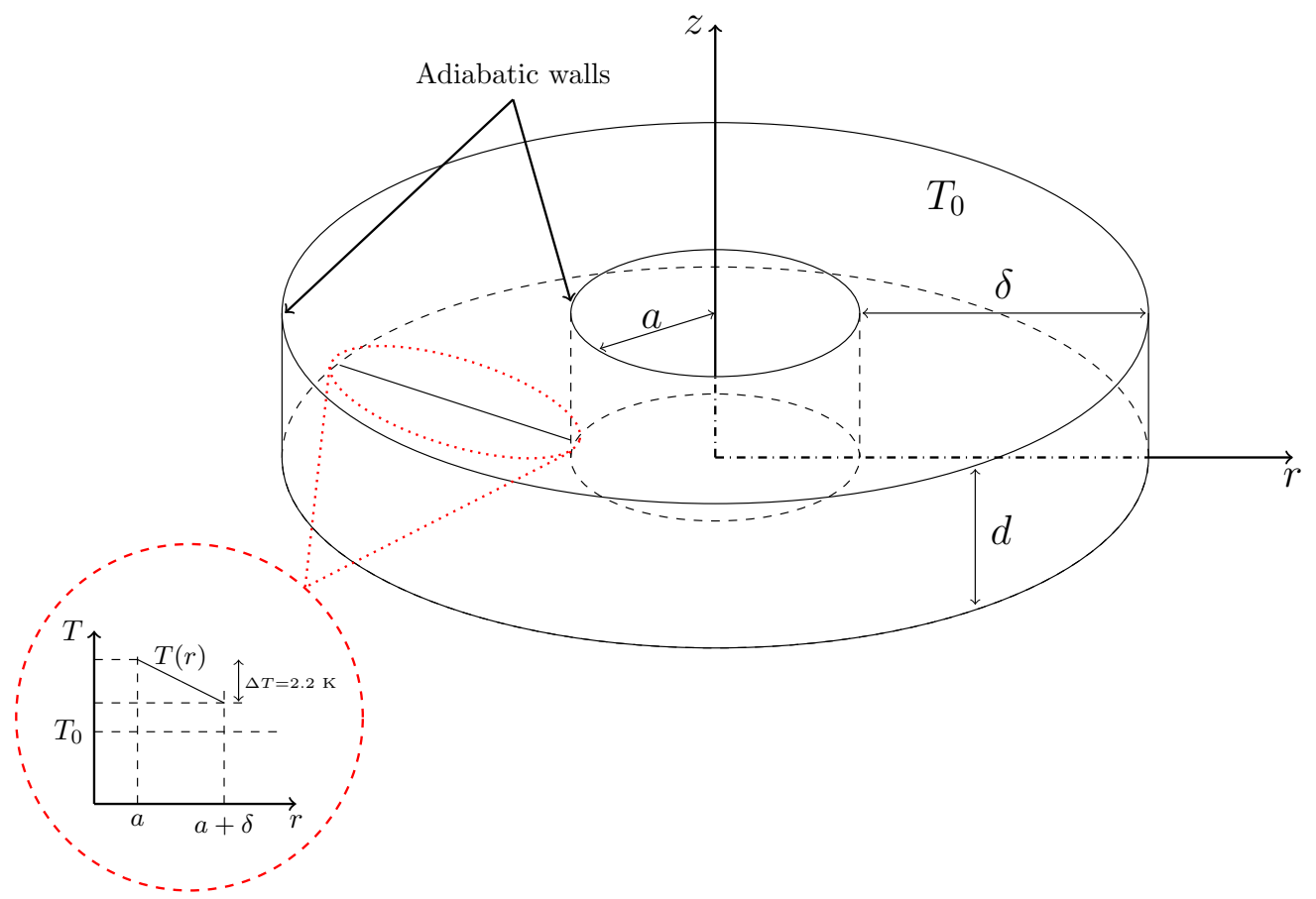

FIG. 1. Sketch of the geometry. Lateral walls are considered adiabatic. The fluid is heated from below and the top surface is open to the atmosphere.

fifth and last section conclusions are presented, and future works are proposed.

\section{COMPUTATIONAL DOMAIN AND PROBLEM FORMULATION}

The physical domain modeled in this work is presented in Fig. 1. A horizontal fluid layer of depth $d$ (the $z$ or axial coordinate) is contained in the space limited by two concentric cylinders of radii $a$ and $a+\delta$ (the $r$ coordinate). In previous works as $[10,16]$, the aspect ratio $\Gamma$ was set to 4 . For the numerical experiments shown here, the aspect ratio $\Gamma=\delta / d$ varies in a range from 1 to 8 . The diameters of the two cylinders are chosen so that the bigger one is double of the smallest one, i.e., $a=\delta$.

A decreasing linear temperature profile from the inner to the outer cylinder was imposed on the bottom wall. The horizontal temperature difference was set to $T_{G}=2.2 \mathrm{~K}$ and remained constant throughout the computations. The wall is considered rigid. The top surface is open to the atmosphere, and the heat transfer to the atmosphere is modeled through the Biot number. The two lateral walls of the cylinder are considered adiabatic. The mean temperature difference between the bottom plate and the atmosphere $\Delta T$ is used as reference temperature in the definition of the Rayleigh and Marangoni numbers.

The system evolves according to the momentum and mass balance equations and to the energy conservation principle, adimensionalized as in [5], i.e.,

$$
\begin{gathered}
\nabla \cdot \mathbf{u}=0, \\
\partial_{t} \mathbf{u}+(\mathbf{u} \cdot \nabla) \mathbf{u}=\operatorname{Pr}\left(-\nabla p+\nabla^{2} \mathbf{u}+\operatorname{Ra} \Theta \mathbf{e}_{z}\right), \\
\partial_{t} \Theta+\mathbf{u} \cdot \nabla \Theta=\nabla^{2} \Theta .
\end{gathered}
$$

In Eqs. (1)-(3), $u_{r}, u_{\theta}$, and $u_{z}$ are the components of the velocity field $\mathbf{u}, \Theta$ is the temperature, and $p$ is the pressure. These are expressed in cylindrical coordinates and $\mathbf{e}_{z}$ is the unit vector in the $z$ direction.

Typically, in order to study these kind of problems, the Boussinesq approximation is used [18]. This approximation states that small horizontal changes in density do not affect the flow and only vertical changes should be considered. This is the origin of the last term in the Navier-Stokes equation [Eq. (2)]. A recent study from Celli et al. [19] shows that the viscous dissipation term in the energy equation (3) might trigger instabilities in some cases. In the current work, several numerical tests have been performed to confirm that the influence of the viscous dissipation term in the present study was minimal so it was finally neglected. Boundary conditions (BCs) are similar to those of Torregrosa et al. [14]. They are summarized in Table I. Briefly, the three velocity components are zero on the cylindrical walls and the bottom plate. In the top surface, the thermocapillarity forces are modeled trough the Marangoni condition [5] and the heat transfer to the atmosphere is modeled by the Biot condition. A temperature gradient is imposed on the bottom plate, while lateral walls are considered adiabatic. In Eqs. (1)-(3) and BCs given by Table I the adimensional numbers introduced previously are used.

TABLE I. Boundary conditions.

\begin{tabular}{lcc}
\hline \hline$z=0$ & $z=d$ & $r=a, a+\delta$ \\
\hline$u_{r}=0$ & $\partial_{z} u_{r}+\mathrm{Ma} \partial_{r} \Theta=0$ & $u_{r}=0$ \\
$u_{\phi}=0$ & $r \partial_{z} u_{\phi}+\mathrm{Ma}_{\phi} \Theta=0$ & $u_{\phi}=0$ \\
$u_{z}=0$ & $u_{z}=0$ & $u_{z}=0$ \\
$\Theta=\Delta T-\left(T_{G} / \delta\right) r$ & $\partial_{z} \Theta+\Theta=0$ & $\partial_{n} \Theta=0$ \\
\hline
\end{tabular}




\section{NUMERICAL METHOD}

As soon as a temperature gradient is imposed, the fluid evolves until a stationary convective motion state is reached. This regime is known as basic state. Since the flow is laminar and due to the symmetries in the problem, the basic state solution can be approximated by a $2 \mathrm{D}$ axisymmetric solution. Therefore, the dependency with the angular coordinate $\phi$ can be neglected. The equations then become

$$
\begin{gathered}
r^{-1} \partial_{r}\left(r u_{r}\right)+\partial_{z} u_{z}=0, \\
\operatorname{Pr}^{-1}\left(u_{r} \partial_{r} u_{r}+u_{z} \partial_{z} u_{r}\right)=-\partial_{r} p+\Delta_{c} u_{r}-\frac{u_{r}}{r^{2}}, \\
\operatorname{Pr}^{-1}\left(u_{r} \partial_{r} u_{z}+u_{z} \partial_{z} u_{z}\right)=-\partial_{z} p+\Delta_{c} u_{z}+\operatorname{Ra} \Theta, \\
u_{r} \partial_{r} \Theta+u_{z} \partial_{z} \Theta=\Delta_{c} \Theta,
\end{gathered}
$$

where $\Delta_{c}=r^{-1} \partial_{r}\left(r \partial_{r}\right)+\partial_{z}^{2}$ is the Laplacian operator expressed in cylindrical coordinates, but simplified due to the previously mentioned symmetries. To obtain the proper solution, the previous equations are supplemented with the boundary conditions presented in Table I.

A collocation method, as the ones presented in the work of Orszag [20], was used to solve the previous system of equations. The procedure starts by expanding the flow variables (pressure, velocity components, and temperature) in a truncated series of orthonormal Chebyshev polynomials, as

$$
\begin{aligned}
& p(r, z) \simeq \sum_{n=0}^{N} \sum_{m=0}^{M} a_{n m} T_{n}(r) T_{m}(z), \\
& u_{r}(r, z) \simeq \sum_{n=0}^{N} \sum_{m=0}^{M} b_{n m} T_{n}(r) T_{m}(z), \\
& u_{z}(r, z) \simeq \sum_{n=0}^{N} \sum_{m=0}^{M} c_{n m} T_{n}(r) T_{m}(z), \\
& \Theta(r, z) \simeq \sum_{n=0}^{N} \sum_{m=0}^{M} d_{n m} T_{n}(r) T_{m}(z),
\end{aligned}
$$

where $T_{i}(x)$ is the Chebyshev polynomial of the first kind of degree $i$. The coefficients $a_{n m}, b_{n m}, c_{n m}$, and $d_{n m}$ of the polynomial series become now the unknowns of the problem. The expanded expressions of the flow variables are substituted into Eqs. (1)-(3) and boundary conditions (Table I). The resultant equations are then evaluated in the Chebyshev-Gauss-Lobatto (CGL) points [21]. CGL points are defined as

$$
\begin{aligned}
& r_{i}=\cos \left(\pi \frac{i}{N}\right), \quad j=0,1, \ldots, N, \\
& z_{i}=\cos \left(\pi \frac{i}{M}\right), \quad j=0,1, \ldots, M,
\end{aligned}
$$

where $N$ and $M$ correspond to the order of the method, i.e., the number of points, in radial and axial direction, respectively. The use of CGL points is of interest when dealing with boundary effects [22] since the points are not equispaced but tend to concentrate near the boundaries, which improves accuracy. The boundary condition for the pressure is obtained projecting the equations by the normal to the boundaries and evaluating the projected equations there. The use of this procedure, as proposed in the work of Mancho et al. [23], avoided the problem of the spurious modes found in other works of the literature as [24]. The pressure is then determined up to an additive constant, an arbitrary value for it is fixed in a boundary point.

A Newton-like iterative method is used to solve the nonlinearity of the problem. To start the iteration either a known basic state "close" to the new one or the solution of the linearized problem [neglecting the nonlinearity of Eqs. (5) and (6)] can be used. Convergence of the iterative procedure was obtained in less than 20 iterations as shown by Hoyas et al. [5].

In all the numerical experiments shown in this paper, a stable basic state is obtained for low Ra. This basic state becomes unstable as $\mathrm{Ra}$ (and thus $\mathrm{Ma}$, for a fixed Bo) is increased. The shape and type of the appearing solution is extremely complex, and all the parameters of the problem play important roles, but in some cases a relationship has been found.

The stability analysis of the basic state is performed based on the technique shown in Hoyas et al. [5]. The procedure consists of introducing a perturbation in the basic state solution with perturbation fields depending on the three coordinates $r, \phi$, and $z$. Since the problem is axisymmetric, and thus there is periodicity in $\phi$, fluid magnitudes may be expanded in Fourier modes in $\phi$ as

$$
X(r, \phi, z, t)=X_{b}(r, z)+X_{p}(r, z) e^{i k \phi+\lambda t},
$$

where the subscripts $b$ and $p$ denote the expanded variable in the basic state and the induced perturbation, respectively; and $k \geqslant 0$ is the wave number and $i=\sqrt{-1}$ is the imaginary unit. It is important to remark again that the basic state does not depend on $\phi$. The linear stability analysis supplies information about the threshold for critical Rayleigh numbers and on the shape of growing instabilities.

The real part of the eigenvalue $\lambda$ characterizes the instability. The perturbation state is stable for $\lambda<0$ and unstable otherwise. In this latter case, the imaginary part of $\lambda$ can be either zero (the bifurcation is stationary) [Fig. 2(a)] or nonzero (the bifurcation is oscillatory) [Fig. 2(b)].

The eigenvalues and eigenfunctions of the problem are computed substituting the Fourier expansion of the different flow variables given in Eq. (12) into the general equations (1)-(3) and the BCs from Table I. Then the unknowns of the problem are the coefficients of the expansion series $\left(a_{n m}, \ldots, d_{n m}\right)$. After using this procedure, the system of equations is linearized by neglecting the nonlinear terms, and thus, a generalized eigenvalue problem is obtained as shown by [14]. The systems becomes

$$
A \bar{X}=\lambda B \bar{X} .
$$

A mesh size convergence study was performed to ensure that the number of points used to discretize the computational domain was enough. Relatively small meshes with 14 in $z$ and 28 points in $r$ are needed to obtain the critical Rayleigh number with eight digit precision.

Imposing the boundary conditions makes the $B$ matrix become singular, and thus not all the eigenvalues have a 


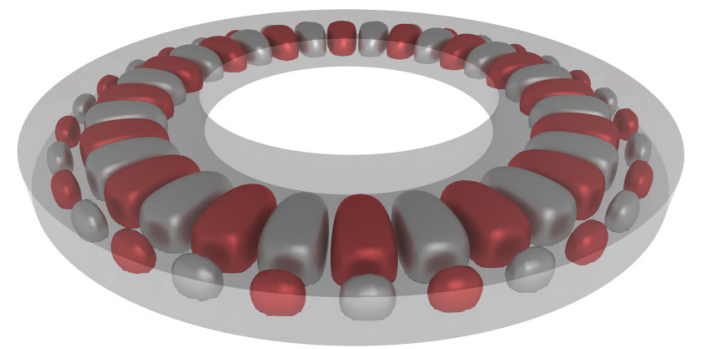

(a)

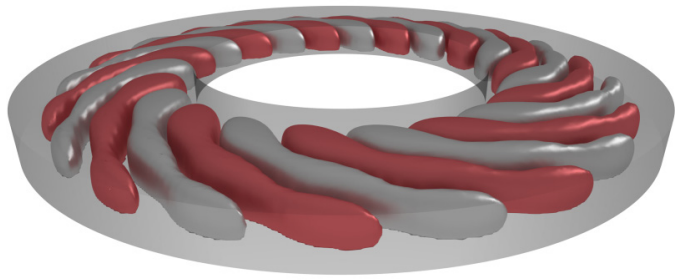

(b)

FIG. 2. Three-dimensional plot of the temperature fields of the growing perturbation: Stationary rolls (top) and hydrothermal waves of first class HWI (bottom).

finite value. To solve this issue, a computational technique specifically developed for thermoconvective problems developed by Navarro et al. [11] is used. The technique consists of a transformation that ensures that the largest eigenvalue obtained in the transformed problem corresponds to the largest finite eigenvalue of the original problem.

Depending on the symmetries of the growing perturbation, several bifurcations may appear, as shown in previous works $[10,16]$. Up to four different competing solutions for the different wave numbers have been found, namely:

(1) Stationary roll (SR), similar to the ones of the basic state [2].

(2) Hydrothermal wave of the first kind or oblique traveling waves (HWI) [2].

(3) Longitudinal rolls (LR) [6].

(4) Standing hydrothermal wave of second class or flowerlike wave (HWII) [3,25].

The code used in the current study has been implemented in Fortran90, based on an earlier implementation developed by Hoyas et al. [5] for infinity Prandtl numbers. The previous code was validated experimentally in [6]. The generalization to $\operatorname{Pr}<50$ was validated in [14], using the same procedure as in [5], and thus ensuring the validity of the results.

\section{DISCUSSION}

In precious studies, several sorts of transitions have been found due to variations in Prandtl number [2] or in aspect ratio [26,27]. However, the influence of the basic state in the instability threshold and the shape of the growing perturbation is not clear yet. A recent publication by Hoyas et al. [16] showed that neither the variation of Biot nor Prandtl numbers affects greatly the shape of the basic state, for a constant value of $\Gamma$. The Bond number was found to be the most significant parameter influencing the onset of instabilities. However,
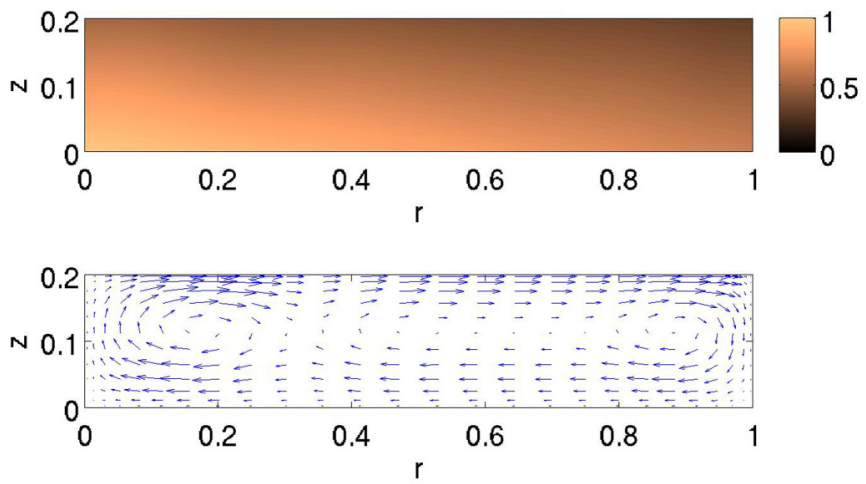

(a)
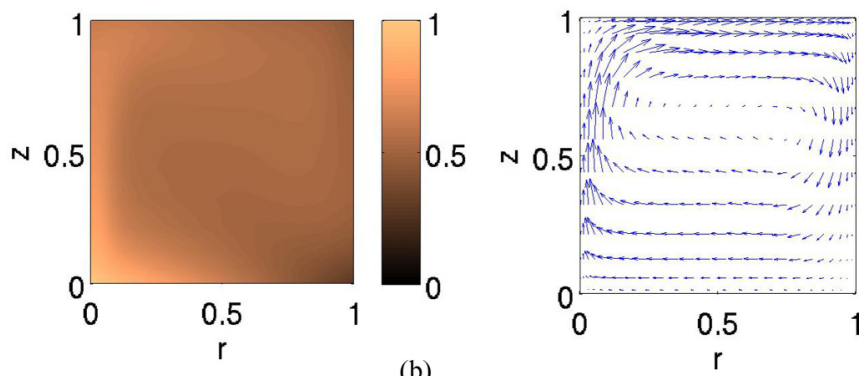

FIG. 3. Representative vertical temperature and velocity fields of the two basic states found in this work: the first one (a) showing several co-rotating rolls and a linear temperature profile (does not present negative temperature gradients); the second one (b) known as return flow presents a single roll, and thus shows regions with negative temperature gradient.

changes in $\Gamma$ are found to have an enormous influence on the basic state, modifying its shape completely.

Two very different basic states have been found. The first one [example given in Fig. 3(a) for the case of $\Gamma=5$ ] presents two or three co-rotating rolls. The temperature field presents a constant decreasing gradient from top to bottom and does not present negative temperature gradients. The temperature field is basically linear, thus is usually named linear flow. For instance, this flow is similar to the one studied in [28] or [10].

The second basic state found is typically known as return flow [see Fig. 3(b) for the particular case of $\Gamma=1$ ]. This regime is characterized by the presence of strong negative vertical temperature gradients. The only whirl of the flow is strong enough to produce a recirculation on the flow. In the central plane of the domain the flow is purely horizontal and therefore is particularly well described by the classical parallel flow approach, which considers $u_{z}=0$. The parallel flow approach has been used in Refs. [2,3,29] to obtain the flow solution in an infinite domain $(\Gamma \rightarrow 0)$. Thus this approach is suitable to describe the flow behavior of the middle plane at small aspect ratios.

Figure 4 shows values of the critical Marangoni number in the $\Gamma$-Bo plane for $\operatorname{Pr}=1.2$. The top-right subfigure indicates the percentage of points in the collocation grid where the temperature gradient is negative. The white line (passing through the origin) separates two very different regions, with a completely different basic state. At the left side of this line the 


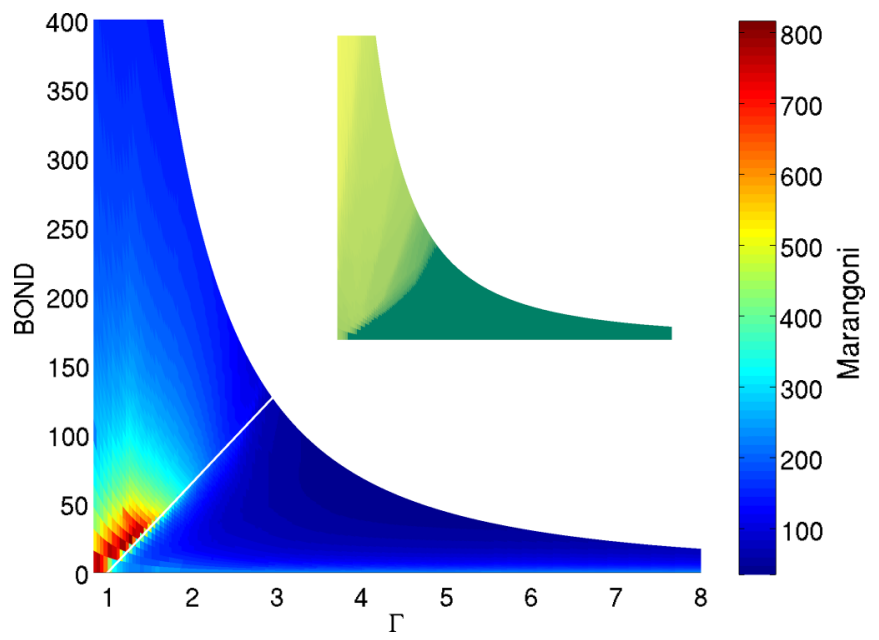

FIG. 4. Marangoni number at the critical temperature. The topright subfigure shows the percentage of points in the grid where the gradient of temperature is negative. The lighter the color, the higher the percentage.

return flow regime, similar to the one presented in Fig. 3(b), appears; while at the right side of the line, the basic state presents the characteristic linear temperature profile shown in Fig. 3(a).

In the cases where a single recirculation (return flow) appears, the critical Rayleigh number (or Marangoni number for a constant Bond) grows drastically, reaching a value two orders of magnitude higher than those for $\Gamma=8$. Even if there is more fluid to store the heat, this drastic increase in Ra can only be explained by the strong swirl appearing in the middle of the cell that enhances the heat transfer across the cylinder.

Representative top $r-\phi$ plane isotherms of the two types of perturbations found in this region (single recirculation basic state) are shown on the top-left corner of Fig. 5 (top), and are explained later. This figure shows the different regions found in the $\Gamma$-Bo plane indicating also the value of the critical wave number $k$ of the perturbation. The curves separating these regions, presented with green dots in the figure, are made up of codimension-two points. In these points, for the same value of the parameters, at least two critical wave numbers, and thus perturbation types, appear. Similarly, the point where two of these curves cross each other is a codimension-three point as obtained in Hoyas et al. [10]. An example of this type of point is located at $\Gamma \approx 3$ and $\mathrm{Bo} \approx 40$. The two regions striped with black curves correspond to cases where a real critical eigenvalue is obtained (stationary bifurcation), and the others correspond to cases with complex eigenvalues (oscillatory bifurcation).

In the region where a linear temperature profile basic state appears (points at the right side of the white line in Fig. 4), the pattern of transitions among the several possible shapes of the growing perturbation is extremely complex. A great deal of transitions, of almost any type, appear. As an example, following the white curve of Fig. 5 (top) from the right to the left, very different perturbations are found, as described below. This curve is such that $g$ is kept fixed $(g=7)$, so Bo $\approx d^{2} \propto \frac{1}{\Gamma^{2}}$ along it. The top subfigure exhibits the different $r-\phi$ plane isotherms for the growing perturbation and the critical wave number in each region, while the bottom one
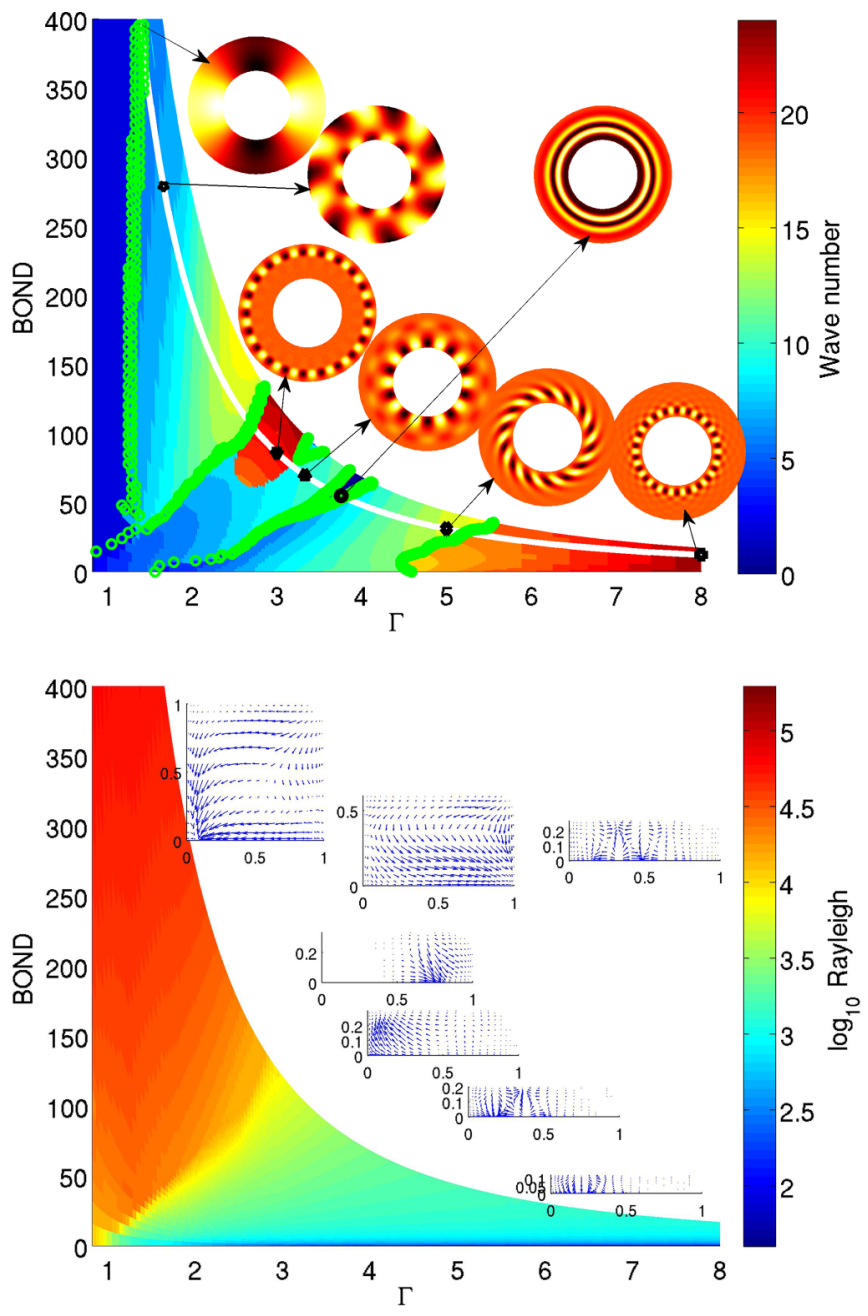

FIG. 5. Top: Critical wave number $k$ in the $\Gamma$-Bo plane. Representative top $r-\phi$ plane isotherms for the growing perturbation at $g=7$ are shown at the top subfigure. Bottom: Critical Rayleigh number (in logarithmic scale) and velocity fields at the same points as in the top figure. Rayleigh number saturates at $2 \times 10^{4}$ to better appreciate the transition region.

shows the corresponding velocity fields. In the isotherms the colors are normalized using the highest absolute temperature as reference value. Lighter colors indicate hot spots while darker ones represent the colder regions.

The rightmost region $(\Gamma \approx 5-8)$ is characterized by a basic state presenting several radial rolls, being the stronger one closer to the hotter (internal) wall. These rolls are concentric with the interior cylinder and are in good agreement with experimental observations shown by Garnier et al. [3]. This is also the place where the stronger swirl appears in the perturbations, as can be observed in the top $r$ - $\phi$ plane isotherm and the velocity diagram. In the second region, from $\Gamma=4$ to $\Gamma=5$, the structure is similar but since a Hopf bifurcation appears, an oscillatory solution is found.

As $\Gamma$ is reduced, the curve enters in a region characterized by a wave number 0 , originating the characteristic concentric longitudinal rolls. This has been found previously by Hoyas et al. [16] but only for very small Biot numbers. The next region shows again radial rolls larger and closer to the internal wall. 
However, this changes completely in the red region $(2.5 \leqslant$ $\Gamma \leqslant 3$ ) where the rolls move to the colder (external) wall, their size is largely reduced, and the wave number is doubled. It is important to point out that the velocity changes accordingly. The former structure of several co-rotaing rolls disappear and only one region with strong swirl can be appreciated. Again, the shape of the growing perturbation of the following region $(\Gamma \leqslant 2.5)$ is completely different. The only reason that can explain this drastic change in the behavior of the flow is the that the basic state is completely modified becoming nonlinear, i.e., with a temperature gradient changing in sign along $r$.

The blue region at the leftmost of Fig. 5 (region of low values $\Gamma$ ) have a value of $k=2$. For any nonzero Bond number, as $\Gamma$ gets smaller, the wave number of the solution $k$ is reduced

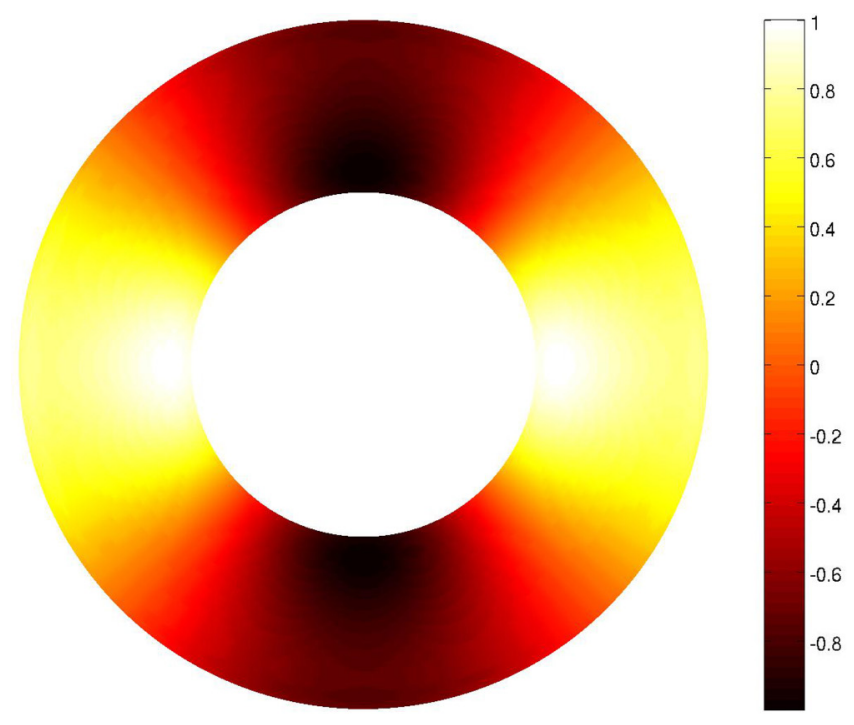

(a)

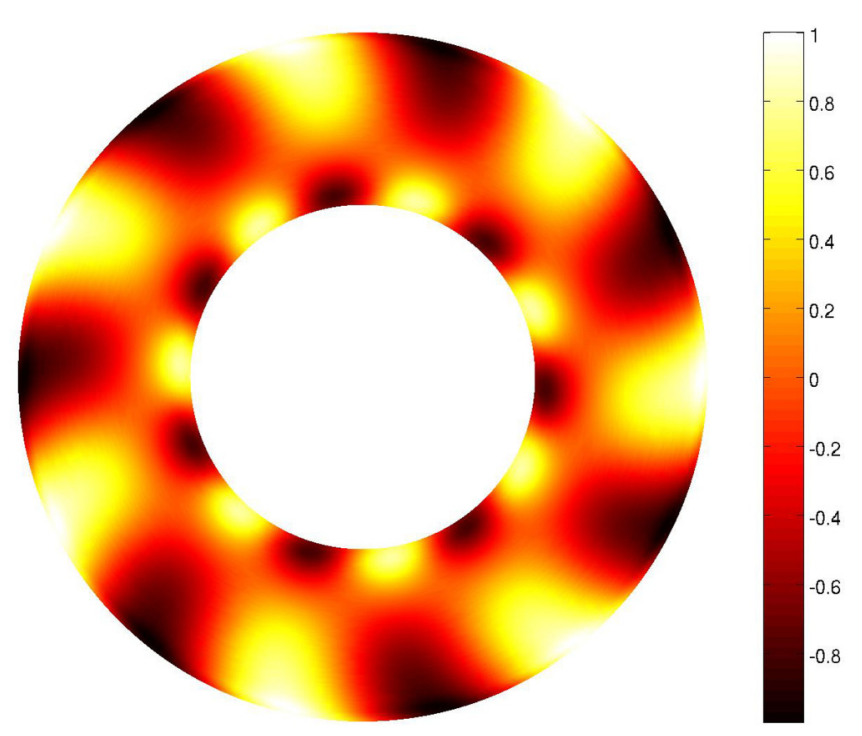

(b)

FIG. 6. Close-up look of the new growing perturbations found in this work. These perturbation are found in the region of low values of $\Gamma$ and their critical wave number $k$ is found to tend to a limit value of 2 . The color scale is normalized using the highest absolute temperature as reference value. tending to the limit value of two. This tendency was found experimentally by [30]. Note that Yu et al. defined the aspect ratio $\epsilon$ as the inverse of the one used in this paper $\Gamma$. The shape of these growing perturbations slightly resembles those found on Refs. [3,6] but to the authors' knowledge this is the first time that hydrothermal waves like these have been found. A close-up look of these new perturbations is shown in Fig. 6.

The lower subfigure of Fig. 5 shows the critical values of the Rayleigh number in the Bo- $\Gamma$ plane. The influence of the basic state is also appreciated in here, at the left side of the white line of Fig. 4, return flow regime, the Rayleigh number is about two orders of magnitude higher than at the right side of the mentioned line (linear regime). To complement the plane isotherms of the top subfigure, the corresponding velocity fields in a vertical plane of each of the growing perturbations found along the constant $g$ line are plotted. Once again a wide variety of different solutions, in this case in terms of the velocity field, coexists is a relatively narrow range of the domain aspect ratio.

Some additional tests, not explicitly included in this paper, were made and it was checked that the critical wave number in this region is only affected by the Prandtl number. It is also important to notice that the Marangoni number in the region close to the left-down corner is larger than in any other place. This means that, in that region, the flow can hold an important surface tension without becoming unstable.

\section{CONCLUSIONS}

This paper assesses the influence of the domain geometry, in particular the aspect ratio $\Gamma$, on the onset of thermoconvective instabilities in a Bérnard-Marangoni convection problem. Numerical flow stability analysis has been performed on an annular domain at a relatively low Prandtl number $(\operatorname{Pr}=1.2)$. The flow is heated from below with an imposed constant linearly decreasing temperature profile. This temperature gradient makes the flow evolve until the basic state is reached. Notice that trying to reproduce this phenomena through experiments requires us to use different fluids to cover a wide range of Bond numbers and each experiment would have typical stabilization times of the range of hours, which indicates the amount of resources required.

The only variable parameters used in this work have been the aspect ratio $\Gamma=\frac{\delta}{d}$ and the Bond number (which basically depends on $g$ and $\Gamma$ ), and Rayleigh number. Two very different basic states have been obtained. The first one presenting several co-rotating rolls, which induce on the flow a quasilinear temperature field (linear flow) with a decreasing gradient from top to bottom. The second one, known as return flow, presents a single recirculation in the domain, inducing a condition in which the flow in the central plane is mainly horizontal, allowing the use of the parallel flow approach to describe the behavior of the flow there. Concerning the perturbations, all the four possible known patterns for the instabilities have been found varying only the aspect ratio (following a constant $g$ line). The four of them are localized in the linear flow region of the $\Gamma$-Bo plane.

In conclusion, the existence of a nonlinear basic state affects the stability in two ways. First, two new perturbations, similar to hydrothermal waves, have been found. Second, the capacity 
of the container to store heat is greatly increased and thus higher critical Rayleigh numbers are needed to unstabilize the flow. On the contrary, the existence of a linear basic state induces a large amount of different perturbations. The physical mechanisms triggering these different solutions are still unknown and they will be the objective pursued by the authors in future investigations.

\section{ACKNOWLEDGMENTS}

The computations shown in this work were made possible by a generous grant of computer time from the supercomputation center of the Universitat Politècnica de València. We are grateful to Mr. José Pedro García-Galache for his help in the preparation of Fig. 2.
[1] H. Bénard, Les tourbillons cellulaires dans une nappe liquide. Méthodes optiques d'observation et d'enregistrement, J. Phys. Theor. Appl. 10, 254 (1901).

[2] M. K. Smith and S. H. Davis, Instabilities of dynamic thermocapillary liquid layer. Part 1. Convective instabilities, J. Fluid Mech. 132, 119 (1983).

[3] N. Garnier and A. Chiffaudel, Two dimensional hydrothermal waves in an extended cylindrical vessel, Eur. Phys. J. B 19, 87 (2001).

[4] S. Hoyas, H. Herrero, and A. Mancho, Bifurcation diversity of dynamic thermocapillary liquid layers, Phys. Rev. E 66, 057301 (2002).

[5] S. Hoyas, H. Herrero, and A. Mancho, Thermal convection in a cylindrical annulus heated laterally, J. Phys. A: Math. Gen. 35, 4067 (2002).

[6] S. Hoyas, A. M. Mancho, H. Herrero, N. Garnier, and A. Chiffaudel, Bénard-Marangoni convection in a differentially heated cylindrical cavity, Phys. Fluids 17, 054104 (2005).

[7] H. Herrero and A. M. Mancho, Influence of aspect ratio in convection due to nonuniform heating, Phys. Rev. E 57, 7336 (1998).

[8] A. M. Mancho, H. Herrero, and J. Burguete, Primary instabilities in convective cells due to nonuniform heating, Phys. Rev. E 56, 2916 (1997).

[9] A. Ezersky, A. Garcimartín, J. Burguete, H. Mancini, and C. Pérez-Garca, Hydrothermal waves in Marangoni convection in a cylindrical container, Phys. Rev. E 47, 1126 (1993).

[10] S. Hoyas, A. Gil, P. Fajardo, and M. Pérez-Quiles, Co-dimension three bifurcations in a Bénard-Marangoni problem, Phys. Rev. E 88, 015001 (2013).

[11] M. C. Navarro, H. Herrero, and S. Hoyas, Chebyshev collocation for optimal control in a thermoconvective flow, Commun. Comput. Phys. 5, 649 (2009).

[12] L. Peng, Y.-R. Li, W.-Y. Shi, and N. Imaishi, Three-dimensional thermocapillary-buoyancy flow of silicone oil in a differentially heated annular pool, Int. J. Heat Mass Transfer 50, 872 (2007).

[13] W. Shi, X. Liu, G. Li, Y.-R. Li, L. Peng, M. Ermakov, and N. Imaishi, Thermocapillary convection instability in shallow annular pools by linear stability analysis, J. Supercond. Novel Magn. 23, 1185 (2010).

[14] A. J. Torregrosa, S. Hoyas, M. J. Pérez-Quiles, and J. M Mompó-Laborda, Bifurcation diversity in an annular pool heated from below: Prandtl and Biot numbers effects, Commun. Comput. Phys. 13, 428 (2013).

[15] E. Eckert, R. Goldstein, W. Ibele, S. Patankar, T. Simon, T. Kuehn, P. Strykowski, K. Tamma, A. Bar-Cohen, J. Heberlein,
J. Davidson, J. Bischof, F. Kulacki, U. Kortshagen, and S. Garrick, Heat transfer-A review of 1997 literature, Int. J. Heat Mass Transfer 43, 2431 (2000).

[16] S. Hoyas, P. Fajardo, A. Gil, and M. Perez-Quiles, Analysis of bifurcations in a Bénard-Marangoni problem: Gravitational effects, Int. J. Heat Mass Transfer 73, 33 (2014).

[17] S. O'Shaughnessy and A. Robinson, Heat transfer near an isolated hemispherical gas bubble: The combined influence of thermocapillarity and buoyancy, Int. J. Heat Mass Transfer 62, 422 (2013).

[18] S. Chandrasekhar, Hydrodynamic and Hydromagnetic Stability (Dover, New York, 1961).

[19] M. Celli, A. Barletta, and L. Alves, Marangoni instability of a liquid film flow with viscous dissipation, Phys. Rev. E 91, 023006 (2015).

[20] S. A. Orszag, Comparison of pseudospectral and spectral approximation, Stud. Appl. Math. 51, 253 (1972).

[21] C. Canuto, M. Hussaini, A. Quarteroni, and T. Zang, Spectral Methods in Fluid Dynamics (Springer, Berlin, 1988).

[22] J. Jiménez and S. Hoyas, Turbulent fluctuations above the buffer layer of wall-bounded flows, J. Fluid Mech. 611, 215 (2008).

[23] A. Mancho and H. Herrero, Instabilities in a laterally heated liquid layer, Phys. Fluids A 12, 1044 (2000).

[24] C. Bernardi and Y. Maday, Approximations Spectrales des Problmes aux Limites Elliptiques (Springer, Berlin, 1992).

[25] E. Favre, L. Blumenfeld, and F. Daviaud, Instabilities of a liquid layer locally heated on its free surface, Phys. Fluids 9, 1473 (1997).

[26] J. Burguete, N. Mukolobwiez, F. Daviaud, N. Garnier, and A. Chiffaudel, Buoyant-thermocapillary instabilities in extended liquid layers subjected to a horizontal temperature gradient, Phys. Fluids 13, 2773 (2001).

[27] F. Daviaud and J. Vince, Traveling waves in a fluid layer subjected to a horizontal temperature gradient, Phys. Rev. E 48, 4432 (1993).

[28] R. Riley and G. Neitzel, Instability of thermocapillary-buoyancy convection in shallow layers. Part 1. Characterization of steady and oscillatory instabilities, J. Fluid Mech. 359, 143 (1998).

[29] J. Mercier and C. Normand, Buoyant-thermocapillary instabilities of differentially heated liquid layers, Phys. Fluids 8, 1433 (1996).

[30] J.-J. Yu, D.-F. Ruan, Y.-R. Li, and J.-C. Chen, Experimental study on thermocapillary convection of binary mixture in a shallow annular pool with radial temperature gradient, Exp. Thermal Fluid Sci. 61, 79 (2015). 